\title{
The effect of the intra-S-phase checkpoint on origins of replication in human cells
}

\author{
Neerja Karnani and Anindya Dutta ${ }^{1}$ \\ Biochemistry and Molecular Genetics, University of Virginia School of Medicine, Charlottesville, Virigina 22908, USA
}

\begin{abstract}
Although many chemotherapy drugs activate the intra-S-phase checkpoint pathway to block S-phase progression, not much is known about how and where the intra-S-phase checkpoint regulates origins of replication in human chromosomes. A genomic analysis of replication in human cells in the presence of hydroxyurea (HU) revealed that only the earliest origins fire, but the forks stall within $2 \mathrm{~kb}$ and neighboring clusters of dormant origins are activated. The initiation events are located near expressed genes with a preference for transcription start and end sites, and when they are located in intergenic regions they are located near regulatory factor-binding regions (RFBR). The activation of clustered neo-origins by HU suggests that there are many potential replication initiation sites in permissive parts of the genome, most of which are not used in a normal $\mathrm{S}$ phase. Consistent with this redundancy, we see multiple sites bound to MCM3 (representative of the helicase) in the region flanking three out of three origins studied in detail. Bypass of the intra-S-phase checkpoint by caffeine activates many new origins in mid- and late-replicating parts of the genome. The intra-S-phase checkpoint suppresses origin firing after the loading of Mcm10, but before the recruitment of Cdc45 and AND-1/CTF4; i.e., after helicase loading but before helicase activation and polymerase loading. Interestingly, Cdc45 recruitment upon checkpoint bypass was accompanied by the restoration of global Cdk2 kinase activity and decrease in both global and origin-bound histone H3 Lys 4 trimethylation (H3K4me3), consistent with the suggestion that both of these factors are important for Cdc45 recruitment.
\end{abstract}

[Keywords: replication origins; hydroxyurea; intra-S-phase checkpoint; pre-RC; Cdc45; genomic mapping]

Supplemental material is available for this article.

Received July 19, 2010; revised version accepted February 2, 2011.

DNA replication is a highly orchestrated process that precisely duplicates the genome once every cell cycle. Prereplicative complexes (pre-RC) are formed on chromatin in late mitosis or early G1 phase to mark potential sites for origin activation (Bell and Dutta 2002; Machida et al. 2005). At the onset of S phase, some of these sites become active origins of replication. Curiously, although $S$ phase is permissive for firing of origins, not all origins fire at the very onset of $S$ phase. Instead, origins are fired in a specific temporal order so that replication of the genome takes well over $8 \mathrm{~h}$ in mammalian cells (Jeon et al. 2005; Karnani et al. 2007, 2010). Perturbations in DNA replication-for example, after treating with chemotherapy drugs-lead to premature stalling of forks and accumulation of damaged DNA (Paulovich and Hartwell 1995; Nyberg et al. 2002; Osborn et al. 2002). Studies in the yeasts have shown that early-replicating origins fire in the presence of such DNA-damaging agents, but origins that normally fire later in $S$ phase are prevented from firing by the activation of the intra-S-phase checkpoint

${ }^{1}$ Corresponding author.

E-MAIL ad8q@virginia.edu; FAX (434) 924-5069.

Article is online at http://www.genesdev.org/cgi/doi/10.1101/gad.2029711.
(Santocanale and Diffley 1998; Shirahige et al. 1998; Dimitrova and Gilbert 2000; Feijoo et al. 2001; Luciani et al. 2004; Merrick et al. 2004; Syljuasen et al. 2005). Similar intra-S-phase checkpoint pathways are active in vertebrates and mammalian cells (Dimitrova and Gilbert 2000; Ben-Yehoyada et al. 2007; Seiler et al. 2007). However, it is not clear exactly which origins fire in the presence of DNA-damaging agents in mammalian cells, and how the other origins are repressed. This is partly because very few origins have been defined, and these origins mostly fire in early $S$ phase. It is also not known whether origins that fire when the checkpoint is bypassed are the same as the origins used in an unperturbed $S$ phase.

Initiation of DNA replication consists of two steps: namely, the formation of the pre-RC (i.e., helicase loading) and activation of a pre-RC (i.e., helicase activation) (Machida et al. 2005; Takeda and Dutta 2005; Hoang et al. 2007; Takara and Bell 2009). This complex is formed during G1 phase of the cell cycle, but its activation occurs at the G1/S transition by S-phase cyclin-dependent kinases (CDKs) and the Cdc7/Dbf4 kinase (DDK), which mediate the association of $\mathrm{Cdc} 45$ with a preformed preRC at each origin (Sclafani and Holzen 2007). In addition to CDK and DDK activities, Mcm10 is required for the 
chromatin binding of Cdc45 (Wohlschlegel et al. 2002; Gregan et al. 2003; Sawyer et al. 2004). In human cells, Mcm10 is recruited to chromatin in G1 phase after the pre-RC formation and remains bound throughout $\mathrm{S}$ phase (Izumi et al. 2001). It interacts with the ORC2 protein and the MCM2-7 complex (Izumi et al. 2000). Mcm10 facilitates replication initiation by recruiting DNA polymerase $\alpha$ at initiation sites in an AND-1-dependent manner (Zhu et al. 2007). Cdk2 kinase activity is required for the loading of both Cdc45 and AND-1. In addition to pre-RC activation, Mcm10 has been proposed to have a role in elongation in budding yeast, as Mcm10 moves with the replication fork from the origin (Kawasaki et al. 2000; Ricke and Bielinsky 2004). Whether Mcm10, Cdc45, and AND1 are loaded at origins upon activation of the intra-S checkpoint is unknown.

Recently we and others generated a catalog of origins by mapping nascent strand (NS) peaks in $1 \%$ of the human genome selected by the ENCODE project to be representative of the whole genome (The ENCODE Project Consortium 2004; Cadoret et al. 2008; Karnani et al. 2010). Interestingly, replication initiates close to transcription start sites (TSSs) and near RNA Pol II-binding sites. In addition, origins were preferentially seeded in the segments of the genome that recruit transcription factors (Karnani et al. 2010). We also delineated which parts of the ENCODE pilot area replicate early or late in S phase (Karnani et al. 2007, 2010). These results set the stage for the current study. We now ask (1) which origins fire and which are repressed in cells treated with the ribonucleotide reductase inhibitor and DNA-damaging agent hydroxyurea (HU)? (2) Is there evidence of active repression of late-firing origins by the intra-S-phase checkpoint? (3) What step in the licensing and activation of origins is inhibited by the checkpoint? (4) Are origin-specific chromatin modifications associated with the checkpoint? Most of our knowledge of the effect of the intra-S-phase checkpoint on origins of replication is derived from experiments in the yeasts and Xenopus egg extracts. Our results verify whether these mechanisms are conserved in human cells, and provide useful insights on the genomic stress caused by $\mathrm{HU}$, a chemotherapeutic drug used in melanomas, chronic myeloid leukemia, metastatic ovarian cancer, polycytheia vera, and sickle cell anemia.

\section{Results}

HU causes activation of multiple, closely spaced initiation sites

HU blocks DNA synthesis by inhibiting the ribonucleotide reductase, thus limiting the pool of deoxynucleotides available for incorporation at replication forks. To identify regions where replication occurs in the presence of $\mathrm{HU}$, we synchronized cells in mitosis by nocodazole treatment and released them into the next cell cycle for $20 \mathrm{~h}$ in the presence of HU and 5-bromo-2'-deoxyuridine (BrdU). Newly replicated DNA incorporating BrdU was immunoprecipitated and hybridized to high-density tiling microarrays (25-mer oligonucleotide probes with an average spacing of 22 base pairs [bp]) representing the nonrepetitive sequence in the 30-Mb ENCODE region (The ENCODE Project Consortium 2004; Karnani et al. 2007, 2010).

Fluorescence-activated cell sorter (FACS) analysis confirmed that the cells synchronously entered S phase after release from mitosis, but that there was not enough DNA synthesis to cause the cells to move significantly beyond the early S-phase population of cells in DNA content (see Fig. 1A; Materials and Methods). Two biological replicates of BrdU-labeled DNA fragments isolated from HUtreated cells were hybridized to independent microarrays (Fig. 1B). To negate the effect of nonspecific binding of genomic DNA to the BrdU antibody, two biological replicates of genomic DNA from unlabeled cells were also immunoprecipitated with the anti-BrdU antibody and hybridized to the arrays as background control samples. Hybridization data were analyzed using the model-based analysis of tiling arrays (MAT) tool (Johnson et al. 2006), and the genomic positions with a statistically significant enrichment $\left(P \leq 10^{-3}\right)$ of BrdU-labeled sites over the control sample in a $1-\mathrm{kb}$ window were flagged as HU-BrIP sites. We identified 178 HU-BrIP sites with a median length of $1.9 \mathrm{~kb}$ (Table 1).

A

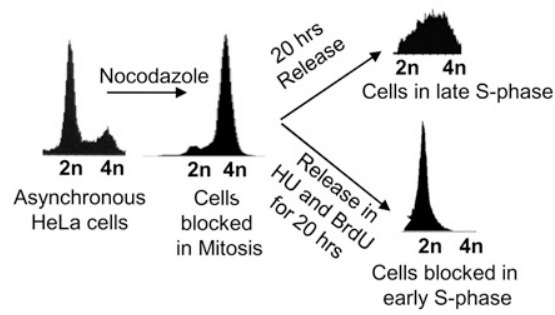

B

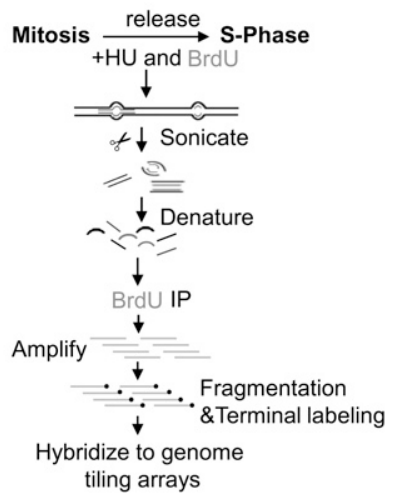

Figure 1. Schematic for HU treatment and sample preparation for microarrays. (A) HeLa cells were synchronized in mitosis by nocodazole treatment for $10 \mathrm{~h}$. After mitotic shake-off, cells were released in either regular medium or medium containing $\mathrm{HU}$ and BrdU. FACS was performed at each step to ensure efficiency of synchronization and HU treatment. $(B)$ BrdUlabeled DNA from HU-treated cells was purified from the total genomic DNA by immunoprecipitation with anti-BrdU antibody. The purified DNA was amplified by PCR, labeled with biotin, and hybridized to tiling arrays. 
Table 1. Summary of initiation sites in $H U$ and after checkpoint abrogation by caffeine in the ENCODE pilot area

\begin{tabular}{lcr}
\hline & $\begin{array}{c}\text { Number of } \\
\text { sites }\left(P=10^{-3}\right)\end{array}$ & $\begin{array}{c}\text { Median } \\
\text { length }\end{array}$ \\
\hline HU-BrIP & 178 & $1892 \mathrm{bp}$ \\
HU-BrIP unique & 44 & $1441 \mathrm{bp}$ \\
HU-BrIP clusters & 134 (distributed & \\
$\quad$ (definition: two or more & within 29 clusters) & \\
$\quad$ sites within 10 kb) & & $11,647 \mathrm{bp}$ \\
HU + caffeine & 310 & $1401 \mathrm{bp}$ \\
CAFSp-BrIP & 186 & $1313 \mathrm{bp}$ \\
\hline
\end{tabular}

The replication timing of these sites was retrieved from our previously published study (The ENCODE Project Consortium 2007; Karnani et al. 2007). BrdU labeling in HU-arrested cells occurred only in the early-replicating parts of the genome, with all of the HU-BrIP sites collectively representing $18 \%$ of the early-replicating ENCODE area. None of the HU-BrIP sites were detected in late-replicating regions of the genome (Fig. 2A). Based on the 1 - to $2-\mathrm{kb} / \mathrm{min}$ fork speed ascertained from the literature (Edenberg and Huberman 1975), the $~ 2-k b-l o n g$ BrdU tracks observed in the presence of HU suggest that forks stall very quickly after origin firing.

An interesting feature of DNA replication in the presence of HU was the extensive clustering of the initiation events. When initiation sites were mapped by BrdU labeling in unperturbed $S$ phase, the median distance between these sites was $16 \mathrm{~kb}$ (Karnani et al. 2010). In contrast, 134 of the $178 \mathrm{HU}-\mathrm{BrIP}$ sites (75\%) were within $10 \mathrm{~kb}$ of their neighbors (Fig. 2B). We classified the HU-BrIP sites into 29 clusters (regions that had two or more sites within $10 \mathrm{~kb}$ ) and 44 unique sites (Table 1). The median intersite distance in the clusters was $\sim 3 \mathrm{~kb}$, significantly smaller than the $16-\mathrm{kb}$ inter-Ori distance in unperturbed $\mathrm{S}$ phase. Most of these clusters (65\%) contained less than four HUBrIP sites (Fig. 2C). The largest cluster, containing 25 sites (spanning $\sim 200 \mathrm{~kb}$ ), was located on chromosome 7 in the region encoding the tumor suppressor locus containing CAV1 and CAV2 genes (Fig. 2D; Williams et al. 2004; Han et al. 2009). This cluster also contains the FRA7G fragile site (Engelman et al. 1998).

Twenty-four percent of HU-BrIP sites overlapped with the NS peaks identified in the normal cell cycle (NS peaks identified by either NS-LExo or NS-BrIP methods in the ENCODE region) (Karnani et al. 2010). The altered pattern of initiation as well as the dense clustering of the HU-BrIP sites suggest the activation of many neighboring dormant origins in the presence of HU. This indicates the tremendous plasticity of initiation site selection in human chromosomes. When fork elongation is perturbed, as in the presence of $\mathrm{HU}$, then these neighboring origins are activated probably in an attempt to continue to replicate the chromosomes. Such dense clustering of potential initiation sites is consistent with the idea that many origins are located in zones of initiation sites (Dijkwel et al. 2002). This observation is also consistent with similar experiments performed on fission yeast (Hayashi et al. 2007), and with molecular combing data from human
A

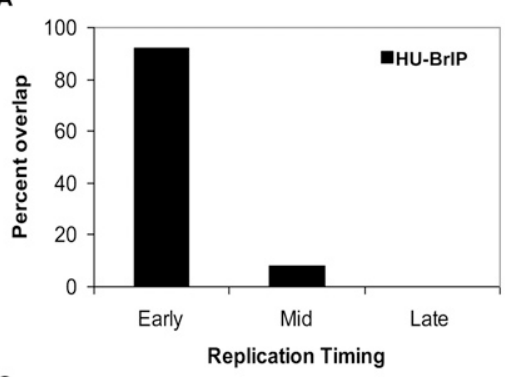

B
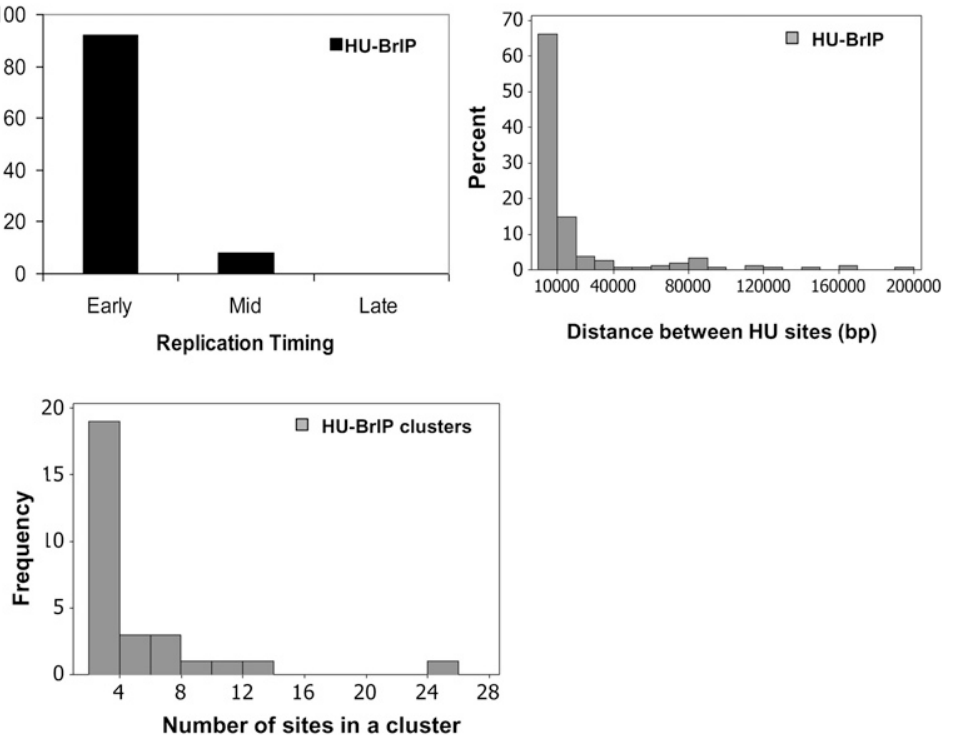

D

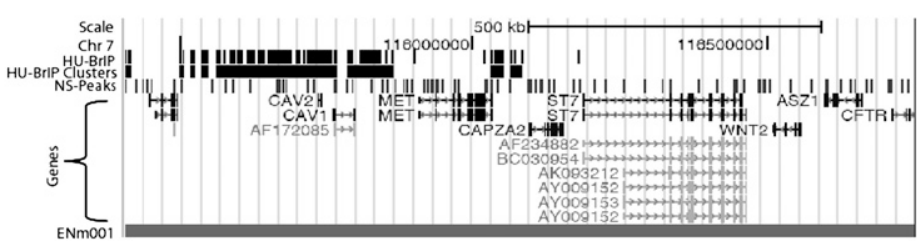

Figure 2. Features of initiation events induced under HU stress. (A) Segregation of HU-BrIP sites by time of replication of the adjoining parts of the chromosomes in a normal S phase (early, mid, and late). (B) Distribution of interorigin distances in $\mathrm{HU}$. The distances between HU-BrIP sites are very small. (C) Clustering of HU-BrIP sites in $10-\mathrm{kb}$ windows. Distribution of the number of HU-BrIP sites per cluster. (D) University of California at Santa Cruz (UCSC) browser image showing intensive clustering of HU-BrIP sites on a 1.3-Mb region of chromosome 7 (ENCODE region ENm001). The browser shot also compares the location of HU-BrIP sites with BrdUlabeled NS peaks from cells in a normal S phase. The genes in the area are also indicated. The "HU-BrIP cluster" track indicates contiguous $10-\mathrm{kb}$ windows with more than two initiation sites. 
U2OS cells (Ge et al. 2007) and an amplified locus in Chinese hamster cells (Anglana et al. 2003; Hayashi et al. 2007). The discovery that many new origins in earlyreplicating parts of the genome are activated in the presence of $\mathrm{HU}$, and yet origins in later-replicating parts of the genome are not activated, suggests that latereplicating origins fail to fire, not because of a shortage of deoxynucleotides, but because they are suppressed by some other mechanism such as an intra-S-phase checkpoint. Conversely, the neo-origins that are activated in the local cluster in the early-replicating part of the genome appear to be immune to the intra-S-phase checkpoint that shuts down initiation at more distal origins.

\section{Inactivation of the intra-S checkpoint by caffeine causes initiation of late origins}

The active suppression of late-firing origins in HU involves the intra-S-phase checkpoint pathway. To investigate this at the genome-wide scale, we inhibited ATR kinase, the enzyme at the apex of the intra-S-phase checkpoint pathway, with caffeine (Sarkaria et al. 1999). HeLa cells synchronized in mitosis with nocodazole either were released for $20 \mathrm{~h}$ into the growth medium containing $\mathrm{HU}+\mathrm{BrdU}$ or were treated with caffeine $+\mathrm{HU}+\mathrm{BrdU}$. Caffeine was added $9 \mathrm{~h}$ after release, when the cells were still in G1, and BrdU-labeled DNA was harvested after an additional $11 \mathrm{~h}$ (total: $20 \mathrm{~h}$ post-release) (Fig. 3A). Activation of intra-S-phase checkpoint in the presence of $\mathrm{HU}$ and its abrogation by addition of caffeine were tested by checking the phosphorylation of Chk1, the target of ATR kinase (Fig. 3B). In parallel, the Cdk2 kinase activity was repressed in HU but elevated in $\mathrm{HU}+$ caffeine (Fig. 3C). Studies in yeast suggest that the intra-S-phase checkpoint strongly suppresses Cdk kinase activity. In contrast, we note only a $50 \%$ decrease in the global kinase activity when the checkpoint is activated.

The FACS profile of caffeine-treated cells still showed them arrested in early S phase (Fig. 3A). There was only a modest increase in total BrdU incorporation in the $\mathrm{HU}+$ caffeine-treated cells compared with HU alone (Supplemental Fig. S1). Since we did not see any change in the FACS profile with caffeine, we next asked whether the modest increase in BrdU incorporation could be detected
A

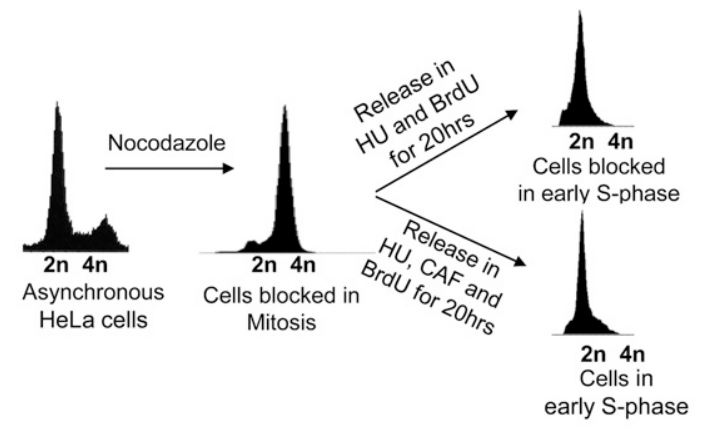

B

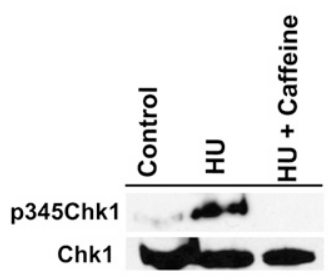

D

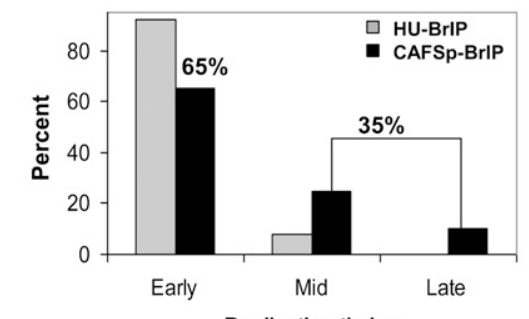

F Replication timing
Figure 3. Effect of checkpoint abrogation on HUinduced replication stress. (A) Cell synchronization and treatment of G1 cells with $\mathrm{HU}+\mathrm{BrdU}$ or $\mathrm{HU}+$ caffeine + BrdU. Propidium iodide FACS of the cells at each stage. $(B)$ Immunoblots of phospho-Chk1 and Chk1 in cells treated as indicated. (C) Total Cdk2 kinase activity measured by in vitro histone $\mathrm{H} 1$ phosphorylation after normalization to the Western signal for Cdk2 in parallel immunoprecipitates. $(D)$ Location of HU-BrIP and CAFSp-BrIP sites based on time of replication in a normal $S$ phase (early, mid, and late). (E) Histogram showing percentage of CAFSp-BrIP sites within 1 or $10 \mathrm{~kb}$ of a HU-BrIP site. (F) UCSC browser image showing location of CAFSp-BrIP sites and HU-BrIP in a $1000-\mathrm{kb}$ region on chromosome 7 (ENCODE region ENm013). Note that some of these CAFSp sites located in earlyreplicating parts of the genome were close to the HU-BrIP cluster. This browser shot also shows initiation in late-replicating parts of the genome upon treatment of HU-arrested cells with caffeine. 
on tiling arrays. BrdU-incorporated DNA isolated from HU + caffeine-treated cells yielded 310 sites, of which 124 overlapped the HU-BrIP sites and 186 were new sites that were activated only in caffeine: caffeine-specific sites (CAFSp-BrIP) (Table 1). The median length of the sites of BrdU incorporation in $\mathrm{HU}+$ caffeine $(1.3 \mathrm{~kb})$ was shorter than the median length in HU alone $(1.9 \mathrm{~kb})$, suggesting that caffeine did not rescue the stalled replication forks and increase elongation. Instead, caffeine promoted the firing of new origins. Thirty-five percent of initiation events in the presence of caffeine occurred in mid- and late-replicating parts of the genome (Fig. 3D), suggesting that abrogation of the checkpoint in the presence of HU initiates late origins. The cells still fail to progress significantly into $\mathrm{S}$ phase due to the lack of dNTPs (Fig. 3A and the limited lengths of BrdU tracks in the CAFSp sites in F). Overall, these results indicate that intra-S-phase checkpoint pathways suppress the firing of origins in the late-replicating parts of the genome in the presence of replication stress.

The remaining $65 \%$ of the CAFSp-BrIP sites were in the early-replicating parts of the genome, allowing us to ask if some of them were created by elongation of forks stalled in HU. Only $3.6 \%$ of CAFSp-BrIP sites, however, were within $1 \mathrm{~kb}$ of HU-BrIP sites, and so could have been created by elongation of forks that stalled in HU (Fig. 3E). An additional $26 \%$ of CAFSp-BrIP sites were within $10 \mathrm{~kb}$ of the HU-BrIP sites, suggesting that these sites could belong to an HU-BrIP cluster (within $10 \mathrm{~kb}$ of the HU-BrIP site) but were independent initiation events (Fig. 3E,F). Just as only $24 \%$ of HU-BrIP sites overlapped with the NS peaks of unperturbed S phase, only $36 \%$ of the caffeineinduced initiation events overlapped with origins from an unperturbed S phase (Karnani et al. 2010). Thus, $64 \%$ of the origins activated upon checkpoint abrogation are de novo initiation sites, again pointing to the plasticity of initiation site selection in human chromosomes.

\section{Origin activity seen in the presence of replication stress occurs preferentially near transcriptional units}

Previous studies have proposed a connection between transcription and replication initiation. For example, origin firing in Xenopus egg extracts can be localized by the assembly of a transcription domain (Hyrien et al. 1995; Danis et al. 2004). In addition, transcription from the DHFR promoter in $\mathrm{CHO}$ cells regulates and defines the boundaries of initiation zones, and, in Drosophila, ORC binding overlaps with RNA Pol II sites (MacAlpine et al. 2004; Saha et al. 2004). Replication origins in HeLa cells preferentially localize at or near RNA Pol II-binding sites and TSSs of genes (Sequeira-Mendes et al. 2009; Karnani et al. 2010). We therefore wondered whether replication under stressed conditions changes the relationship between transcription and replication.

Sixty-five percent of the HU-BrIP sites overlapped with genes $(P<0.02)$, and $94 \%$ of these genes were expressed in HeLa cells (Fig. 4A). A third of these sites were at or
A

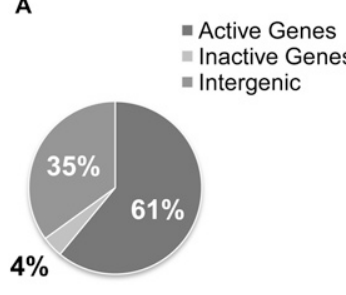

HU-BrIP

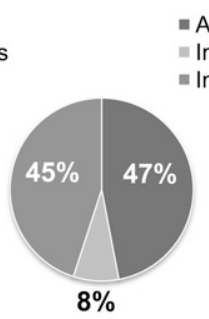

CAFSp-Early - Active Genes
- Inactive Genes - Intergenic
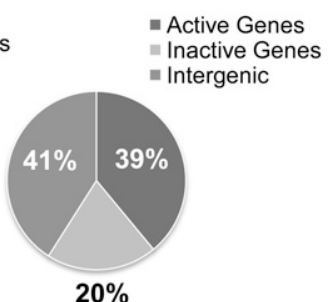

CAFSp-Mid\&Late C

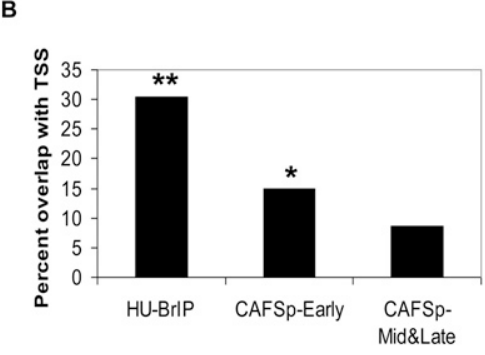

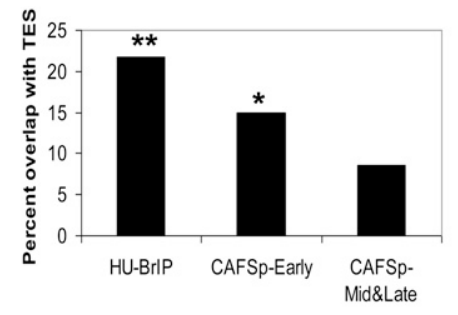

Figure 4. Correlation of HU-BrIP sites and CAFSpBrIP sites with expressed genes and transcription factor-binding regions. $(A)$ Pie chart showing percent distribution of HU-BrIP sites and CAFSp-BrIP sites (segmented by time of normal replication) in the genic and intergenic regions of the genome. The genes are further divided into transcriptionally active and inactive genes. $(B-D)$ Correlation of HU-BrIP, early CAFSp-BrIP, and mid and late CAFSp-BrIP with TSSs, TESs, and RFBRs. Significant enrichment relative to random model at $P<0.01\left(^{*}\right)$ and $P<0.0001\left(^{\star *}\right)$ is indicated.

D

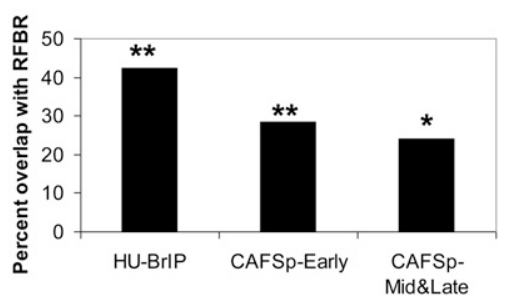


within $1 \mathrm{~kb}$ of the TSS $(P<0.0001)$, and an additional $22 \%$ $(P<0.0001)$ were within $1 \mathrm{~kb}$ of the transcription end sites (TESs) of these expressed genes (Fig. 4B,C). Concordant with this, a third of the HU-BrIP sites also overlapped RNA Pol II-binding sites $(P<0.0001)$. These findings suggest that, even in the presence of suboptimal replication conditions, origins that fire have some preference for transcription units.

We also checked whether abrogation of the checkpoint activates replication origins that are near transcription units. Fifty-five percent of the early-replicating CAFSpBrIP sites and $59 \%$ of mid- and late-replicating CAFSpBrIP sites overlapped with genes (Fig. 4A). Eighty-five percent $(P<0.0001)$ of the genes associated with early CAFSp-BrIP were expressed (Fig. 4A). Since mid- and latereplicating parts of the genome have fewer expressed genes, a lower percentage of mid and late CAFSp-BrIP sites $(66 \%)$ were near transcribing genes, but origins were still enriched near the transcribed genes relative to the random distribution $(P<0.0001)$ (Fig. 4A). However, the association of mid and late CAFSp-BrIP sites with RNA Pol IIbound sites dropped to $14 \%$, and only a small percentage $(<16 \%)$ were localized at TSSs or TESs (Fig. 4C,D).

Previously, we showed that replication origins also display an affinity for the regulatory factor-binding regions (RFBRs) of the genome (RFBRs contain both activator- and repressor-binding sites) (Karnani et al. 2010). The open chromatin surrounding these regions is most likely conducive for recruitment and activation of replication proteins. This correlation persisted with the intergenic HU-BrIP sites, as $40 \%$ overlapped with the RFBR clusters $(P<0.0001)$ (Fig. 4D). More than a quarter of the intergenic CAFSp-BrIP sites were also near these regulatory clusters $(P<0.005)$.

In summary, the origins that are activated when cells are forced to replicate in HU or after abrogation of the intra-S-phase checkpoint are still preferentially located near the start or end of transcriptionally active genes or near RFBRs.

Origins suppressed by the intra-S-phase checkpoint are blocked after Mcm10 but before Cdc45 and AND-1 recruitment

In human cells, Mcm10 binds to chromatin after formation of pre-RCs in the G1 phase of the cell cycle. Mcm10 is involved in origin activation by facilitating Cdc45 loading, and is also required for loading of DNA polymerase $\alpha$ in an AND-1-dependent manner. We first studied the binding of Mcm10 and AND-1 at CAFSp-BrIP sites, origins that were repressed by the intra-S-phase checkpoint in $\mathrm{HU}$ and derepressed when the checkpoint was relieved by caffeine (HUCAF).

Mcm10 and AND-1 chromatin immunoprecipitations (ChIPs) were performed from the chromatin of HU- or $\mathrm{HU}+$ caffeine-treated cells, and five CAFSp-BrIP peaks and five negative control regions were tested for the chromatin loading of these two replication factors. AND-1 binding was stimulated in four out of five CAFSp-BrIP sites when caffeine was added, suggesting that AND-1 recruitment corresponds to origin firing (Fig. 5A). We failed to see AND-1 enrichment in four of the five negative control regions. One negative control region recruited AND-1, but the recruitment was higher in HU compared with $\mathrm{HU}+$ caffeine. This site could represent a site with inefficient initiation in $\mathrm{HU}$ that was not captured in the microarray experiment.

In contrast, Mcm10 was bound to CAFSp-BrIP sites in $\mathrm{HU}$ (Fig. 5B). Binding of Mcm 10 to sites that do not fire in the presence of $\mathrm{HU}$ is consistent with the assembly of a pre-RC complex at these sites, marking them as potential origins of replication. Interestingly, Mcm10 appeared to be lost from these sites when the origins were activated by caffeine. This observation can be explained by either Mcm10 coming off the chromatin after replication initiation or it moving with the replication fork away from a fired origin.

Previous studies have shown that Mcm10 stays on the chromatin throughout S phase (Izumi et al. 2001), so we planned to test the second possibility by walking along the chromosomes on either side of three independent CAFSp-BrIP sites (CAFSp1, CAFSp3, and CAFSp6). These

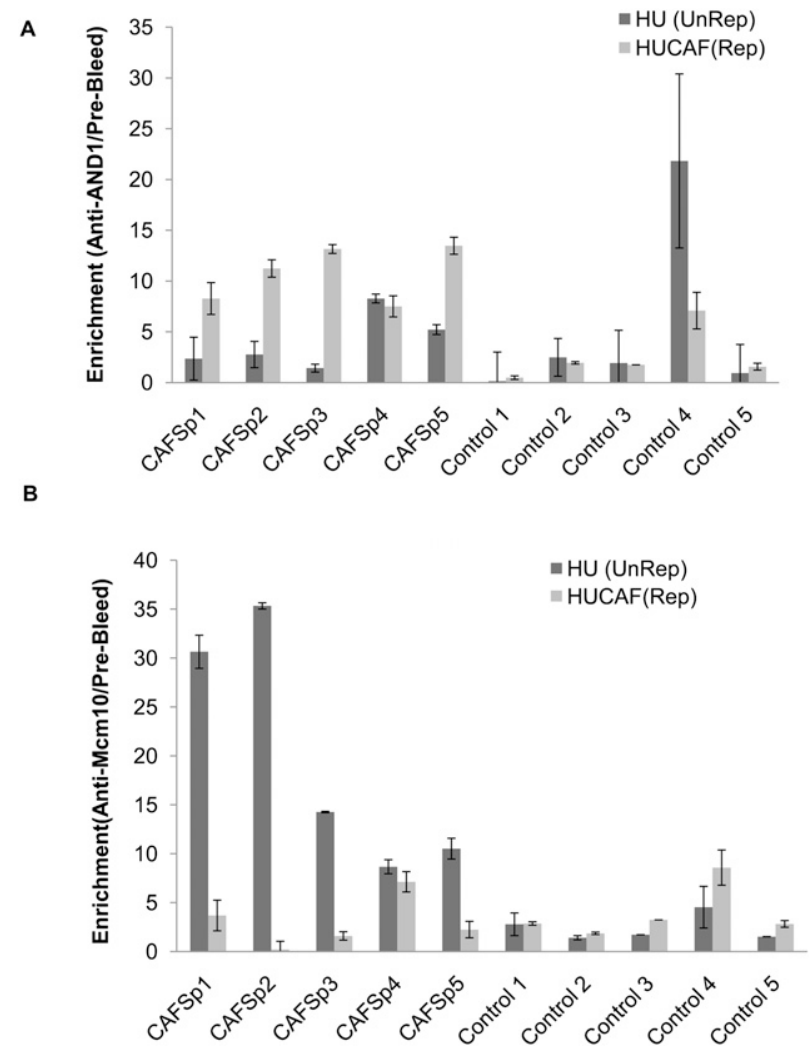

Figure 5. Mcm10 and AND-1 loading on CAFSp-BrIP sites. Five CAFSp-BrIP peaks (two early-, two mid-, and one latereplicating) and five negative control regions were tested for AND-1 $(A)$ and Mcm10 $(B)$ loading on chromatin by ChIP assays from HU (when CAFSp-BrIP sites are unreplicated) and +caffeine (when these sites initiate replication) conditions. Prebleed was used as a measure of nonspecific chromatin precipitation. Quantitative PCR (qPCR) was used to ascertain fold enrichment over prebleed on the tested genomic regions. 
three sites were picked because it was easy to design primers at evenly spaced distances due to the low abundance of repeats around them. We first ascertained the site of pre-RC assembly by performing ChIP with ORC4 and MCM3 antibodies. In the presence of HU, ORC4 was associated with the CAFSp3 origin (Fig. 6A), and also the CAFSp1 and CAFSp6 origins, where it was offset from the BrdU-labeled region by $0.75-1.25 \mathrm{~kb}$ (Supplemental Figs. S3A, S4A). MCM3 was also loaded on all three origins in $\mathrm{HU}$, although MCM3 was loaded not only at the ORCbound site in CAFSp1 and CAFSp6, but at additional sites within $3 \mathrm{~kb}$ (Fig. 6B; Supplemental Figs. S3B, S4B), consistent with the idea that MCM2-7 moves away from the loading site, allowing for additional rounds of MCM2-7 loading (Remus et al. 2009). In addition, an extended walk around CAFSp3 revealed additional MCM3-binding sites in the unreplicated/HU condition that overlapped with ORC4 loading (Supplemental Fig. S2A,B). Therefore, multiple peaks of MCM3 loading may be created by a single
ORC (as in CAFSp1 and CAFSp6) or by multiple ORCs bound to adjoining sites (CAFSp3). These additional loaded molecules of MCM may randomly initiate in a cell population, creating an initiation zone, or not fire at all (as here), creating dormant origins that fire as neo-origins under conditions of replication stress. The important point is that Mcm10, MCM2-7, and ORC were all bound at or near the licensed origins before they fired and when the checkpoint was active.

Upon treatment with caffeine, when the origins have fired, both ORC4 and MCM3 binding at the origins was decreased (Fig. 6A,B; Supplemental Figs. S3, S4). The decrease in ORC4 suggests that ORC is dissociated from fired origins. We initially thought that the decrease in MCM3 was because it moved away from the initiation site as part of the replication fork. However, an extended walk of $20 \mathrm{~kb}$ around CAFSp3 did not detect peaks of MCM3 in HUCAF that were absent in HU, because either the forks stall at variable sites in the cell population so
A

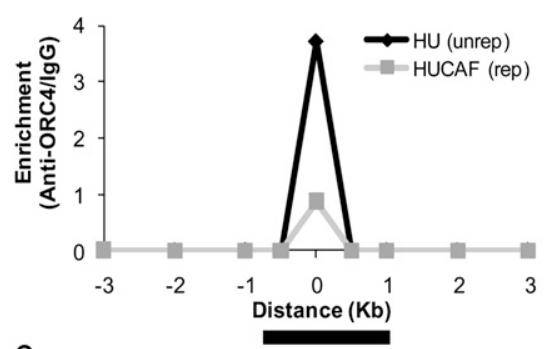

C

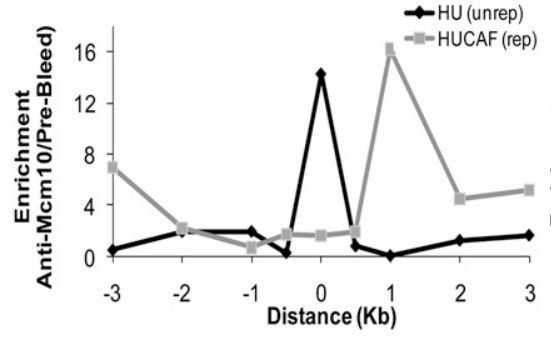

E

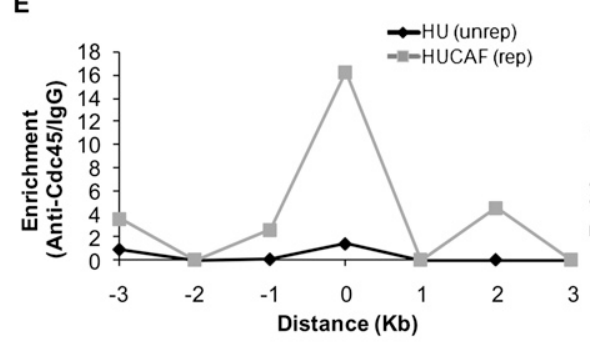

G (i)

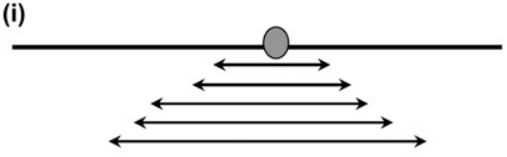

$\longrightarrow$ Origin
$\longleftrightarrow \begin{aligned} & \text { Replicated DNA and } \\ & \text { fork migration }\end{aligned}$

\section{B}

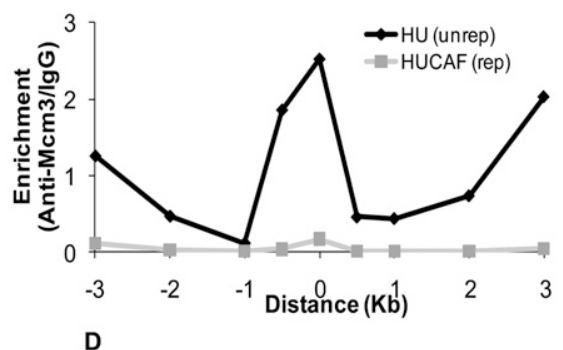

D

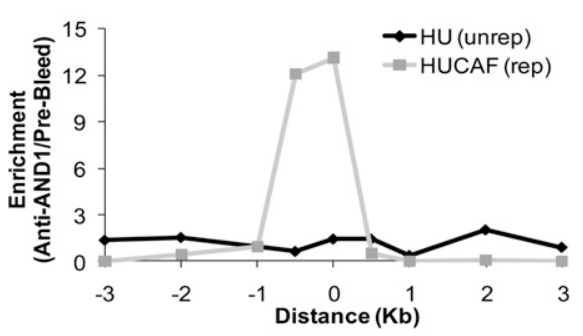

$\mathbf{F}$

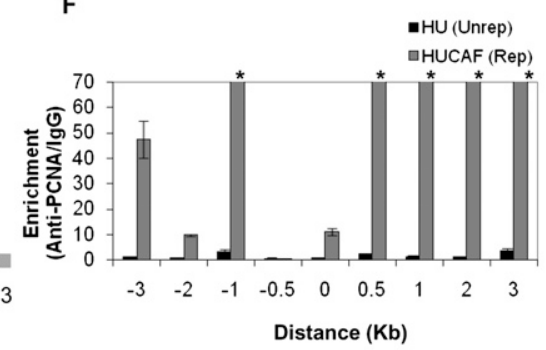

G (ii)

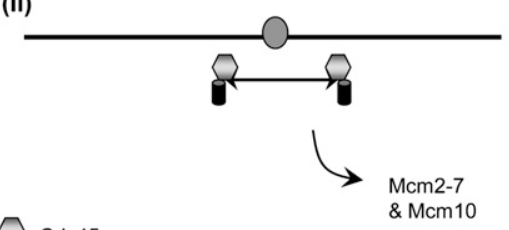

Figure 6. ChIP assay to analyze the distribution of replication factors before and after replication initiation at a CAFSp origin. $(A-F)$ Primers were designed to check the binding of ORC4, MCM3, Mcm10, AND-1, Cdc45, and PCNA at and within $3 \mathrm{~kb}$ of the CAFSp3-BrIP peak (selected from CAFSpBrIP sites used in Fig. 5). Chromatin association of the replication machinery with these sites was checked in the presence of $\mathrm{HU}$ (when these sites did not initiate replication: HU [Unrep]) as well as HU + caffeine (when these sites did replicate: HUCAF [Rep]) conditions. qPCR was used to ascertain fold enrichment over prebleed or IgG on the tested genomic regions. The 0 on the $X$-axis in each graph indicates the peak position of the BrdU signal, while the scale represents the distance of qPCR primers from the peak. The black bar below the $X$-axis in $A$ indicates the length of the population-averaged BrdU-labeled track identified on the microarrays. $\left({ }^{\star}\right)>70 .(G$, panel $i)$ A model showing initiation of some origins with stalling of forks at varied sites under replication stress. These give rise to BrdU and PCNA tracks, but the factors that move with the fork do not pile up at specific flanking sites. (Panel ii) In addition, the same origin in other cells may not fire or fire only minimally despite having recruited Cdc45 and AND-1. The forks stalled by the lack of dNTPs could lose their helicase due to the absence of the stabilizing checkpoint kinase, while retaining Cdc45 and AND- 1 at a site very close to the origin. 
that there is insufficient signal at any one point (see model in Fig. 6G, panel i), or the MCM2-7 complex is actively dissociated from even unfired or minimally fired origins when the checkpoint is bypassed in the continued presence of $\mathrm{HU}$ (see below when discussing AND-1 and Cdc45; Fig. 6G, panel ii).

We next compared Mcm10 loading with respect to the pre-RC. At CAFSp1, Mcm10 moved away from the origin on exposure to caffeine, while at the other two origins, the Mcm10 signal diminished over the whole area (Fig. 6C; Supplemental Figs. S3C, S4C). As with MCM2-7, the disappearance of Mcm10 may be a function of forks stalling at multiple sites or the helicase being disassembled off the chromosomes, while the translocated peak of Mcm10 in CAFsp3 may be atypical and reflect the dissociation of the Mcm10 from the rest of the helicase complex.

In contrast to ORC4, MCM3, and Mcm10, AND-1 and Cdc45 were loaded on the origin only when caffeine bypassed the checkpoint (Fig. 6D,E; Supplemental Figs. S3D,E, S4D,E). Thus, activation of the intra-S-phase checkpoint in human cells suppresses origins after Mcm10 loading but before Cdc45 and AND-1 recruitment, while bypass of the checkpoint allowed Cdc45 and AND-1 recruitment. The location of the Cdc45 and AND-1 peaks near the centers of the CAFSp origins (Fig. 6D,E; Fig. $\mathrm{S} 3 \mathrm{D}, \mathrm{E})$ was surprising, because we had expected them to move with the replication forks like MCM3 and Mcm10.

To resolve this paradox, we delineated the extent over which replication has proceeded by investigating PCNA loading in and around CAFSp3 in HU and HUCAF (Fig. $6 \mathrm{~F})$. PCNA is required for both leading and lagging strand synthesis and is left behind on the newly replicated DNA even after the ligation of Okazaki fragments (Shibahara and Stillman 1999; Sporbert et al. 2002). Thus, chromatin-associated PCNA serves as a marker of newly replicated DNA, at least until the PCNA rings are actively unloaded by Replication Factor C (RF-C). Indeed, PCNA was not associated with the locus in HU (unreplicated) and was easily detected at multiple sites over $6 \mathrm{~kb}$ around CAFSp3 in HUCAF (replicated). PCNA was present on a longer extent of DNA than the averaged BrdU track detected on the microarray, suggesting that PCNA ChIP was a more sensitive assay for recent DNA replication than BrdU immunoprecipitation and microarray hybridization. Clearly, DNA replication had proceeded beyond $3 \mathrm{~kb}$ on either side of CAFSp3 in HUCAF, and yet Cdc45 and AND-1 were stranded near the center of this origin.

The minimal hypothesis for explaining these results is that assays for replication (BrdU incorporation and PCNA loading) score positive even if a subset of the CAFSp3 origins in the cell population fires in the presence of caffeine. The Cdc45 and AND-1 peaks seen when caffeine is added, however, are derived from CAFSp3 loci that have recruited these proteins when the checkpoint is bypassed, but have either not fired or had the forks stall almost immediately due to the lack of dNTPs. The absence of MCM3 in these peaks at unfired (or minimally fired) origins is explained by dissociation of the MCM2-7 complex from the DNA when the checkpoint is inacti- vated under conditions that are still inimical for replication due to the continued presence of HU.

\section{Chromatin status upon activation and after abrogation of the intra-S-phase checkpoint}

Post-translational modification of histones plays an important role in many nuclear processes, including DNA replication. Histone $\mathrm{H} 3$ Lys 4 trimethylation (H3K4me3) has been shown recently to play an important role in the intra-S-phase checkpoint (Liu et al. 2010). MLL, a H3K4 methyltransferase, is degraded by $\mathrm{SCF}^{\text {skp2 }}$ E3 ligase in normal $\mathrm{S}$ phase, but is stabilized by ATR-mediated phosphorylation upon induction of replication stress by HU. The stable form of MLL was proposed to induce H3K4me3 at late-S-phase origins to inhibit Cdc45 loading and delay replication. Liu et al. (2010) studied the change in $\mathrm{H} 3$ methylation on activation of the intra-S-phase checkpoint by studying a single late-firing origin-that at the $\beta$ globin locus. Since we developed an inventory of origins that is repressed by the intra-S-phase checkpoint, we can now ask whether H3K4me3 is similarly implicated in multiple origins.

To investigate this, we first checked whether checkpoint activation and bypass had a global effect on total H3K4me3 levels in the cell (Fig. 7A). In comparison with normal S-phase cells, addition of HU dramatically increased the total H3K4me3 pools. This extensive methylation was significantly alleviated when caffeine was added. We next tested whether this global change in methylation of H3K4 was seen at the checkpoint-arrested replication origins by performing $\mathrm{H} 3 \mathrm{~K} 4 \mathrm{me} 3 \mathrm{ChIP}$ on seven CAFSp-BrIP sites from segments of the genome that normally replicate in early, mid, or late $S$ phase. Three of the seven sites showed an increase in $\mathrm{H} 3 \mathrm{~K} 4 \mathrm{me} 3$ in HU compared with normal S phase. The failure to see a significant increase in $\mathrm{H} 3 \mathrm{~K} 4 \mathrm{me} 3$ in four out of seven checkpoint-arrested origins suggests that such an increase cannot be the sole mechanism by which Cdc45 loading is prevented at all origins. This is especially true for CAFSp1, where H3K4me3 did not increase in $\mathrm{HU}$ (when Cdc45 is not bound) and is paradoxically increased in caffeine (when Cdc45 is recruited). Despite this, it is noteworthy that $\mathrm{H} 3 \mathrm{~K} 4 \mathrm{me} 3$ decreased upon checkpoint bypass at five out of seven of the origins, concurrent with Cdc45 recruitment. Thus, H3K4me3 may indeed be a barrier to Cdc45 loading at origins, and the global increase in this modification upon checkpoint activation probably contributes to blocking Cdc45 recruitment at many (but not all) checkpoint-suppressed origins.

\section{Discussion}

We studied the replication events that occur in the presence of HU and following abrogation of the checkpoint activated by $\mathrm{HU}$ in human cells. A large number of dormant origins in early-replicating parts of the genome are activated in the presence of HU along with active suppression of origins in late-replicating parts of the genome by the intra-S-phase checkpoint. Upon abrogation of the checkpoint by caffeine, many more dormant 
A

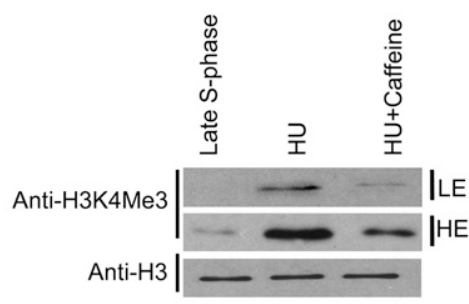

B

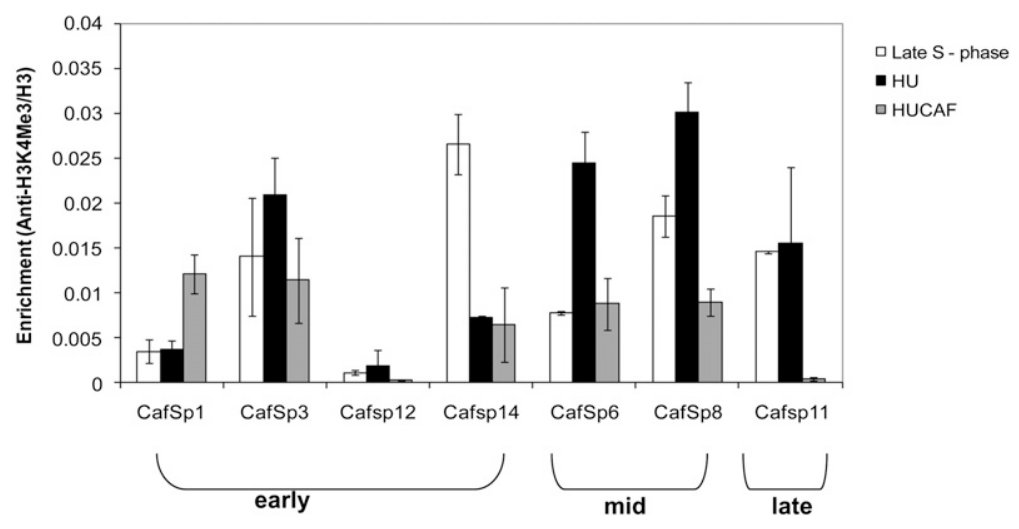

Figure 7. Changes in histone methylation on activation and abrogation of the intra-S-phase checkpoint. HeLa cells synchronized in mitosis with nocodazole were released for $20 \mathrm{~h}$ into either the growth medium or the media containing $\mathrm{HU}$ or $\mathrm{HU}+$ caffeine as detailed in Figure 3. Cells from each of these conditions were processed for either immunoblotting or ChIP assay. (A) Immunoblot showing total $\mathrm{H} 3 \mathrm{~K} 4 \mathrm{me} 3$ levels and histone $\mathrm{H} 3$ as loading control. (LE) Lower exposure; (HE) higher exposure. (B) ChIP assay was performed to check the status of $\mathrm{H} 3 \mathrm{~K} 4 \mathrm{me} 3$ at four early-, two mid-, and one late-S-phase CAFSp sites. origins are activated, not only in early-replicating parts, but notably in late-replicating parts of the genome. The dormant origins that are activated in both the presence and absence of the intra-S-phase checkpoint are mostly independent from origins used during the normal S phase, but share the common feature of preferential location at or near transcriptionally active genes or RFBRs. This may reflect an affinity of the dormant origins for open chromatin. Finally, we show that the intra-S-phase checkpoint pathways suppress origin firing after Mcm10 loading but before Cdc45 and AND-1 recruitment-steps that are dependent on cyclin-Cdk kinase activity and blocked by excess $\mathrm{H} 3 \mathrm{~K} 4 \mathrm{me} 3$. Override of the checkpoint is accompanied by the recruitment of Cdc45 and AND-1 to the origins, concurrent with the restoration of Cdk2 kinase activity and decrease of $\mathrm{H} 3 \mathrm{~K} 4 \mathrm{me} 3$. The concurrent decrease in MCM3 and Mcm10 on the originproximal DNA could be because of migration of these proteins with replication forks that stall at multiple points in the locus. However, the persistence of other fork proteins (Cdc45 and AND-1) at the origins in the checkpoint-bypassed cells leads us to suspect that (1) the BrdU and PCNA tracks are created by only a subset of the chromosomes in the population firing when the checkpoint is bypassed, (2) Cdc45 and AND-1 persist at the significant subset of unfired origins, and (3) MCM3 and $\mathrm{Mcm} 10$ are disassembled from these unfired origins once the checkpoint is bypassed under conditions that are still unfavorable to DNA synthesis. Together, these results yield a comprehensive view of how origins of replication in mammalian cells are regulated by the intraS-phase checkpoint, and point to the tremendous plasticity of initiation site selection in human cells.
Experiments in both budding and fission yeast have shown that, when two origins are located close together, it is rare that both origins are active in the same cell during S phase (Brewer and Fangman 1993; Dubey et al. 1994; Marahrens and Stillman 1994). This "origin interference" is not related to pre-RC assembly, as these factors have been shown to bind efficiently to all origins (Santocanale et al. 1999). An alternative explanation for this phenomenon is that these closely spaced origins might not fire at the same time, and forks from the first origin might pass through the second origin, rendering it inactive. Our observations suggest that, in the presence of $\mathrm{HU}$, forks initiated from the primary origins stall within a short distance, giving an opportunity for the neighboring dormant origins to fire, accounting for the clustering of the new origins. These findings are in agreement with the DNA fiber studies in $\mathrm{CHO}$ and U2OS human cells, where multiple initiations occur on a given DNA molecule when cells are subjected to replication inhibitors (Anglana et al. 2003; Ge et al. 2007). Dormant origin firing is facilitated by the excessive loading of pre- $\mathrm{RC}$ components, specifically the MCM2-7 helicase complex, during G1 phase of the cell cycle. We can see evidence of this "excess" MCM2-7 loading in the multiple peaks of MCM3 around CAFSp1, CAFSp3, and CAFSp6 even before any DNA replication.

Checkpoint activation is known to inhibit firing of late origins (Santocanale and Diffley 1998; Shirahige et al. 1998; Dimitrova and Gilbert 2000; Feijoo et al. 2001; Luciani et al. 2004; Merrick et al. 2004; Syljuasen et al. 2005). Abrogation of the checkpoint reveals that replication in late-replicating parts of the genome can be restored, although many more sites in early-replicating 
parts are also activated. However, most of these initiations also occurred at dormant origins that are not used in the unperturbed cell cycle. Although BrdU-labeled tracks were generated from these newly fired CAFSp origins, we believe that a significant number of origins recruited Cdc45 and AND-1 but did not fire, allowing these proteins to be detected as discrete peaks within the CAFSp origins. We believe that the replication forks fired in caffeine still stall due to the lack of dNTPs, although this can also arise as a consequence of incomplete checkpoint abrogation. Sucrose gradient studies from HeLa cells have shown that DNA strands synthesized in the presence of caffeine are smaller than those made in its absence. This phenotype is more associated with the checkpoint and not caffeine because similar observations are also made upon knockdown of Chk1 (Lehmann 1972; Ge et al. 2007). Despite the use of a different genome-wide approach with genome-tiling arrays, our results are in agreement with these studies, and suggest that relief of the checkpoint did not restore elongation to stalled forks but stimulated the firing of dormant origins of replication. This is not because the intra-S-phase checkpoint does not inhibit fork elongation, but most likely is because the override of the checkpoint by caffeine is unable to restore the collapsed forks.

We found that the intra-S-phase checkpoint suppresses origin firing at some stage between Mcm10 loading and Cdc45/AND-1 recruitment. In Xenopus egg extracts, CDK activity is necessary after Mcm 10 loading at origins and before Cdc 45 or AND-1 recruitment. This leads us to suspect that repression of the Cdk2 kinase by the intraS-phase checkpoint in mammalian cells is responsible for the suppression of CAFSp origins. It is worth noting, however, that the Cdk2 kinase that we measured was not completely repressed in $\mathrm{HU}$ (Fig. 3C). The complete failure to load Cdc45 and AND-1, therefore, must be due to additional reasons. Possible reasons include an undiscovered mechanism to repress Cdk2 kinase locally in the vicinity of the CAFSp origins (by local kinases, phosphatases, or Cdk inhibitors), or the increase in H3K4me3 seen at many (but not all) checkpoint-suppressed origins.

The ORC4 loading at both the CAFSp-BrIP sites was dramatically reduced after initiation of replication, suggesting that ORC may be removed from the chromatin after replication initiation. The removal of DNA-bound ORC could play a role in the actual firing of origins, or more likely is the result of the passage of the replication fork through DNA bound by ORC. ORC1 has been reported to dissociate from chromatin in S phase (Li and DePamphilis 2002; Mendez et al. 2002), but this result suggests that similar dissociation may also occur for the other ORC subunits. We also show that the MCM2-7 complex (1) can decouple from ORC even before replication initiation to slide to variable lengths along the DNA, giving rise to the multiple peaks of MCM3 around the CAFSp origins in HU, and (2) is loaded at greater stoichiometry relative to ORC. This is consistent with recent studies in Xenopus and Saccharomyces cerevisiae (Pacek et al. 2006; Remus et al. 2009; Tsakraklides and Bell 2010). As discussed before, this "excess" MCM2-7, may account for the "excess" of potential origins and the plasticity of initiation sites. The dissociation of the helicase from unfired origins that still retain Cdc45 and AND-1 is an unexpected response of the cell to the unusual stress of checkpoint bypass under conditions that are still bad for DNA replication. The intra-S-phase checkpoint maintains the stability of stalled replication forks in yeast (Lopes et al. 2001), so our result may simply be a variation of this phenomenon. When the checkpoint is bypassed by caffeine, the activation of the helicase through recruitment of Cdc45 may lead to many of the forks stalling at or very close to the origin due to the lack of dNTPs. In the absence of the checkpoint kinases, these stalled forks apparently lose their helicase and thus become destabilized.

Multiple origins fire during $S$ phase, and these events prefer the open chromatin regions of the genome available around TSSs, RNA Pol II-binding sites, and RFBRs. We found this to be true for the dormant origins activated under replication stress. Interestingly, even the mid- and late-firing origins that initiated in the presence of caffeine were located within the bodies of expressed genes.

A striking observation was the global increase of $\mathrm{H} 3 \mathrm{~K} 4 \mathrm{me} 3$ levels in $\mathrm{HU}$ and reduction of the same upon addition of caffeine. This is entirely consistent with the proposed stabilization of MLL by the checkpoint (Liu et al. 2010). By ChIP assay, this induction in HU was recapitulated on three out of seven CAFsp sites, and all of these plus an additional two demonstrated a decrease in H3K4me3 upon checkpoint bypass by caffeine. Incidentally, the H3K4me3 induced was not restricted to the site of Cdc45 loading, but extended throughout the 6-kb region where tested (CAFSp3, CAFSp6, CAFSp8, and CAFSp11) (data not shown). Taken together, these results support the hypothesis that an increase of $\mathrm{H} 3 \mathrm{~K} 4 \mathrm{me} 3$ may account for failure to load Cdc45 at some, but not all, checkpoint-arrested origins.

\section{Materials and methods \\ Cell synchronization and treatment of cells with $\mathrm{HU}$ and caffeine}

HeLa cells at $60 \%$ confluence were treated with $40 \mathrm{ng} / \mathrm{mL}$ nocodazole for $10 \mathrm{~h}$ to arrest them in metaphase. After mitotic shake-off and three washes with PBS, the nocodazole-arrested cells were released into medium containing $1 \mathrm{mM}$ HU with or without $100 \mu \mathrm{M}$ BrdU for $20 \mathrm{~h}$. For caffeine $(5 \mathrm{mM})$ treatment, cells were released from nocodazole into $\mathrm{HU}+\mathrm{BrdU}$ as mentioned above, and, $9 \mathrm{~h}$ post-release, the medium was replaced with $\mathrm{HU}+$ caffeine + BrdU-containing medium. Cells were incubated in this medium for $11 \mathrm{~h}$ (total of $20 \mathrm{~h}$ post-release). Synchronized and treated cells were either used for genomic DNA extraction or fixed in $70 \%$ ethanol and stained with propidium iodide (PI) for FACS by standard methods.

\section{Sample preparation and hybridization to microarrays}

Genomic DNA extracted from HU-treated cells was sonicated to 0.8 - to 1 -kb fragments and subjected to anti-BrdU-IP, amplification, labeling, and hybridization (Karnani et al. 2010). DNA 
recovered after anti-BrdU-IP from cells that were not treated with BrdU was hybridized as the control.

To identify origins of replication, ENCODE01-Forward $(\mathrm{P} / \mathrm{N}$ 900543; Affymetrix) tiling arrays were used as described previously (Karnani et al. 2007). Each microarray was scanned and analyzed for signal intensities using the GeneChIP Scanner 3000 and GeneChIP Operating Software (GCOS) (Affymetrix). The primary data in the form of .CEL files can be accessed at http:// genome.bioch.virginia.edu/HU. All of the data were processed using the hg17 build (NCBI Build 35, May 2004) of the human genome assembly and can be accessed at http://genome.bioch. virginia.edu/HU.

\section{Identification of sites replicated in the presence of $\mathrm{HU}$} and $\mathrm{HU}+$ caffeine

Replication sites were identified by estimating the significance of the enrichment of signal in the treatment sample (BrdUIP-DNA from BrdU-labeled cells) over that in the control sample (BrdU-IP-DNA from unlabeled cells) in a sliding window of 1000 bp using MAT. Two biologically independent treatment and control samples were used for this analysis. Chromosomal regions that showed signal enrichment in the treatment over the control at $P \leq 0.001$ were flagged as HU-BrIP for HU-treated cells and HUCAF for HU + caffeine-treated cells. The HUCAF-BrIP sites that did not overlap HU-BrIP sites and were specifically induced by caffeine treatment were called CAFSp-BrIP sites.

\section{Calculation of inter-HU-BrIP and inter-CAFSp-BrIP distances}

The HU-BrIP and CAFSp-BrIP sites were segregated into the 44 ENCODE regions. For each ENCODE region containing $x$ number of sites, $x-1$ number of distances were calculated, ignoring the distances on the outer sides of the outermost sites in each ENCODE region. Distances from all ENCODE regions were pooled and plotted as a histogram, with the $X$-axis binning distances between replication sites and the $Y$-axis giving the frequency of the number of distances that fell into each bin.

\section{Identifying clusters of initiation}

An initiation cluster is defined as a genomic region that contains two or more HU-BrIP sites within $10 \mathrm{~kb}$. The algorithm used for this analysis calculates the distance between the adjoining endpoints of two adjacent sites. If the endpoints of the two BrdU sites fall within $10 \mathrm{~kb}$ of each other, the two sites are scored as part of a cluster. The total length of the cluster is determined by the distance between the chromosomal start position of the first site and the chromosomal end position of the last site in the cluster.

\section{Comparative analysis with other genomic features}

Data sets for other genomic features such as genes, RNA Pol II, TSSs, TESs, DNaseI-hypersensitive sites, and RFBR clusters were obtained from the University of California at Santa Cruz genome browser (The ENCODE Consortium, http://genome. ucsc.edu/ENCODE). For comparative analysis, HU-BrIP and CAFSp-BrIP sites were treated as the source data set, and the genomic feature to which it was compared was referred to as the target data set. Each BrdU-labeled site in the source set was compared against the target set to check if it overlapped the target site. The percentage of source sites that overlapped with target sites was determined and plotted. To test the significance of this overlap, a random model was generated for each source/target comparison by randomizing the source sites within the ENCODE universe. The source/target analysis described above was repeated for this randomized source set to the fixed target set. This randomization was iterated 9999 times to produce a distribution of the percentage of source sites that overlapped with target sites in 10,000 trials. A $P$-value was ascertained by calculating the fraction of trials that yielded a higher percentage of overlaps than the experimental source set. For example, a $P$-value of 0.0005 indicates that there is a 5 in 10,000 probability that, by random chance, one would identify an overlap higher than the experimental overlap.

\section{ChIP}

ChIP assay was performed as described (Trinklein et al. 2004). Samples were sonicated $(20$ cycles of 30 -sec pulses at $50 \%$ amplitude and $30 \mathrm{sec}$ of cooling on ice) using a Branson microtip $(3.2 \mathrm{~mm})$ and Fisher Model 500 Sonic Dismembrator. The antibodies used for ChIP recognize Mcm10 (BWH128), AND-1, and ORC4 (BWH 66), and have been validated extensively previously (Dhar et al. 2001; Wohlschlegel et al. 2002; Zhu et al. 2007). The MCM3, Histone H3, and H3K4me3 antibodies were purchased from Abcam, and PCNA and Cdc45 antibodies were from Santa Cruz Biotechnology, Inc. Two microliters of ChIP DNA was used as template for quantitative PCR. DNA from ChIP with either preimmune sera or IgG was used as a negative control. Primer details for the ChIP assay are available on request. The CAFsp1, CAFsp3, and CAFsp6 origins were chosen for the chromosomal walks because the paucity of repeat sequences around these sites allowed us to design specific primers to sample evenly spaced sites in the walk. The negative control regions in Figure 5 were selected randomly with the following considerations: (1) These genomic regions did not show BrdU incorporation in the microarray hybridization data, and (2) the primers designed in these regions gave a single amplicon.

\section{Immunoblotting and kinase assay}

For Western blotting, cells were lysed in IPH buffer $150 \mathrm{mM}$ Tris- $\mathrm{HCl}$ at $\mathrm{pH} 8.0,150 \mathrm{mM} \mathrm{NaCl}, 5 \mathrm{mM}$ EDTA, $0.5 \%$ [vol/vol] NP-40, $1 \mathrm{mM}$ dithiothreitol, $20 \mathrm{mM} \mathrm{NaF}$, protease inhibitor mix [Sigma]). Equal amounts of cell lysates were resolved on SDS-PAGE, and the proteins were transferred to the membrane and blotted with rabbit anti-phospho-Chk1 (Ser-345) (Cell Signaling Technology), rabbit anti-Chk1 (Novus Biologicals), mouse anti-H3K4me3 (Millipore), and rabbit anti-Histone H3 (Abcam) antibodies.

For kinase assay, cells were treated with lysis buffer $(50 \mathrm{mM}$, Tris- $\mathrm{HCl}$ at $\mathrm{pH} 7.4,0.2 \%$ Nonidet $\mathrm{P} 40,150 \mathrm{mMNaCl}, 1 \mathrm{mM}$ EDTA, protease inhibitors) supplemented with $50 \mathrm{mM} \mathrm{NaF}$ and $1 \mathrm{mM} \mathrm{Na}_{3} \mathrm{VO}_{4}$. Equal amounts of lysates (1000 $\mu \mathrm{g}$ of protein) were immunoprecipitated with anti-Cdk2 antibody (Santa Cruz Biotechnology). The pellet was washed three times in lysis buffer and twice in kinase wash buffer $150 \mathrm{mM}$ Tris- $\mathrm{HCl}$ at $\mathrm{pH} 7.4$, $10 \mathrm{mM} \mathrm{MgCl}_{2}, 5 \mathrm{mM} \mathrm{MnCl}_{2}$ ), and was divided in two parts. One part was resuspended in $2 \times$ sample buffer, and the second half was resuspended in $20 \mu \mathrm{L}$ of kinase reaction buffer $(50 \mathrm{mM}$ Tris$\mathrm{HCl}$ at $\mathrm{pH} 7.4,10 \mathrm{mM} \mathrm{MgCl}_{2}, 5 \mathrm{mM}$ dithiothreitol, $10 \mu \mathrm{M}$ ATP, $0.5 \mu \mathrm{Ci}$ of $\left[\gamma_{-}{ }^{2} \mathrm{P}\right] \mathrm{ATP}, 2 \mu \mathrm{g}$ of histone $\left.\mathrm{H} 1\right)$. The kinase reaction mixture was incubated for $30 \mathrm{~min}$ at $30^{\circ} \mathrm{C}$, and the reaction was stopped by the addition of equal volume of $2 \times$ sample buffer. Results were analyzed by separating the products by SDS-PAGE and autoradiography.

\section{Acknowledgments}

We thank the Dutta laboratory members for helpful discussions. This work was supported by the National Institutes of Health Grants CA89406 and CA60499 to A.D. 


\section{References}

Anglana M, Apiou F, Bensimon A, Debatisse M. 2003. Dynamics of DNA replication in mammalian somatic cells: nucleotide pool modulates origin choice and interorigin spacing. Cell 114: 385-394.

Bell SP, Dutta A. 2002. DNA replication in eukaryotic cells. Annu Rev Biochem 71: 333-374.

Ben-Yehoyada M, Gautier J, Dupre A. 2007. The DNA damage response during an unperturbed S-phase. DNA Repair (Amst) 6: 914-922.

Brewer BJ, Fangman WL. 1993. Initiation at closely spaced replication origins in a yeast chromosome. Science 262: 1728-1731.

Cadoret JC, Meisch F, Hassan-Zadeh V, Luyten I, Guillet C, Duret L, Quesneville H, Prioleau MN. 2008. Genome-wide studies highlight indirect links between human replication origins and gene regulation. Proc Natl Acad Sci 105: 1583715842.

Danis E, Brodolin K, Menut S, Maiorano D, Girard-Reydet C, Mechali M. 2004. Specification of a DNA replication origin by a transcription complex. Nat Cell Biol 6: 721-730.

Dhar SK, Delmolino L, Dutta A. 2001. Architecture of the human origin recognition complex. J Biol Chem 276: 2906729071.

Dijkwel PA, Wang S, Hamlin JL. 2002. Initiation sites are distributed at frequent intervals in the Chinese hamster dihydrofolate reductase origin of replication but are used with very different efficiencies. Mol Cell Biol 22: 3053-3065.

Dimitrova DS, Gilbert DM. 2000. Temporally coordinated assembly and disassembly of replication factories in the absence of DNA synthesis. Nat Cell Biol 2: 686-694.

Dubey DD, Zhu J, Carlson DL, Sharma K, Huberman JA. 1994. Three ARS elements contribute to the ura4 replication origin region in the fission yeast, Schizosaccharomyces pombe. EMBO J 13: 3638-3647.

Edenberg HJ, Huberman JA. 1975. Eukaryotic chromosome replication. Annu Rev Genet 9: 245-284.

The ENCODE Project Consortium. 2004. The ENCODE (ENCyclopedia Of DNA Elements) project. Science 306: 636-640.

The ENCODE Project Consortium 2007. Identification and analysis of functional elements in $1 \%$ of the human genome by the ENCODE pilot project. Nature 447: 799-816.

Engelman JA, Zhang XL, Lisanti MP. 1998. Genes encoding human caveolin-1 and -2 are co-localized to the D7S522 locus (7q31.1), a known fragile site (FRA7G) that is frequently deleted in human cancers. FEBS Lett 436: 403-410.

Feijoo C, Hall-Jackson C, Wu R, Jenkins D, Leitch J, Gilbert DM, Smythe C. 2001. Activation of mammalian Chk1 during DNA replication arrest: a role for Chk1 in the intra-S phase checkpoint monitoring replication origin firing. I Cell Biol 154: 913-923.

Ge XQ, Jackson DA, Blow JJ. 2007. Dormant origins licensed by excess Mcm2-7 are required for human cells to survive replicative stress. Genes Dev 21: 3331-3341.

Gregan J, Lindner K, Brimage L, Franklin R, Namdar M, Hart EA, Aves SJ, Kearsey SE. 2003. Fission yeast Cdc23/Mcm 10 functions after pre-replicative complex formation to promote Cdc45 chromatin binding. Mol Biol Cell 14: 3876-3887.

Han F, Gu D, Chen Q, Zhu H. 2009. Caveolin-1 acts as a tumor suppressor by down-regulating epidermal growth factor receptor-mitogen-activated protein kinase signaling pathway in pancreatic carcinoma cell lines. Pancreas 38: 766-774.

Hayashi M, Katou Y, Itoh T, Tazumi A, Yamada Y, Takahashi T, Nakagawa T, Shirahige K, Masukata H. 2007. Genome-wide localization of pre-RC sites and identification of replication origins in fission yeast. $E M B O /$ 26: 2821.

Hoang ML, Leon RP, Pessoa-Brandao L, Hunt S, Raghuraman MK, Fangman WL, Brewer BJ, Sclafani RA. 2007. Structural changes in Mcm5 protein bypass Cdc7-Dbf4 function and reduce replication origin efficiency in Saccharomyces cerevisiae. Mol Cell Biol 27: 7594-7602.

Hyrien O, Maric C, Mechali M. 1995. Transition in specification of embryonic metazoan DNA replication origins. Science 270: 994-997.

Izumi M, Yanagi K, Mizuno T, Yokoi M, Kawasaki Y, Moon KY, Hurwitz J, Yatagai F, Hanaoka F. 2000. The human homolog of Saccharomyces cerevisiae Mcm10 interacts with replication factors and dissociates from nuclease-resistant nuclear structures in G(2) phase. Nucleic Acids Res 28: 47694777.

Izumi M, Yatagai F, Hanaoka F. 2001. Cell cycle-dependent proteolysis and phosphorylation of human Mcm10. I Biol Chem 276: 48526-48531.

Jeon Y, Bekiranov S, Karnani N, Kapranov P, Ghosh S, MacAlpine D, Lee C, Hwang DS, Gingeras TR, Dutta A. 2005. Temporal profile of replication of human chromosomes. Proc Natl Acad Sci 102: 6419-6424.

Johnson WE, Li W, Meyer CA, Gottardo R, Carroll JS, Brown M, Liu XS. 2006. Model-based analysis of tiling-arrays for ChIPchip. Proc Natl Acad Sci 103: 12457-12462.

Karnani N, Taylor CM, Malhotra A, Dutta A. 2007. Pan-S replication patterns and chromosomal domains defined by genome tiling arrays of ENCODE genomic areas. Genome Res 17: 865-876.

Karnani N, Taylor CM, Malhotra A, Dutta A. 2010. Genomic study of replication initiation in human chromosomes reveals the influence of transcription regulation and chromatin structure on origin selection. Mol Biol Cell 21: 393-404.

Kawasaki Y, Hiraga S, Sugino A. 2000. Interactions between $\mathrm{Mcm} 10 \mathrm{p}$ and other replication factors are required for proper initiation and elongation of chromosomal DNA replication in Saccharomyces cerevisiae. Genes Cells 5: 975-989.

Lehmann AR. 1972. Effect of caffeine on DNA synthesis in mammalian cells. Biophys J 12: 1316-1325.

Li CJ, DePamphilis ML. 2002. Mammalian Orc1 protein is selectively released from chromatin and ubiquitinated during the S-to-M transition in the cell division cycle. Mol Cell Biol 22: 105-116.

Liu H, Takeda S, Kumar R, Westergard TD, Brown EJ, Pandita TK, Cheng EH, Hsieh JJ. 2010. Phosphorylation of MLL by ATR is required for execution of mammalian S-phase checkpoint. Nature 467: 343-346.

Lopes M, Cotta-Ramusino C, Pellicioli A, Liberi G, Plevani P, Muzi-Falconi M, Newlon CS, Foiani M. 2001. The DNA replication checkpoint response stabilizes stalled replication forks. Nature 412: 557-561.

Luciani MG, Oehlmann M, Blow JJ. 2004. Characterization of a novel ATR-dependent, Chk1-independent, intra-S-phase checkpoint that suppresses initiation of replication in Xenopus. J Cell Sci 117: 6019-6030.

MacAlpine DM, Rodriguez HK, Bell SP. 2004. Coordination of replication and transcription along a Drosophila chromosome. Genes Dev 18: 3094-3105.

Machida YJ, Hamlin JL, Dutta A. 2005. Right place, right time, and only once: replication initiation in metazoans. Cell 123: $13-24$.

Marahrens Y, Stillman B. 1994. Replicator dominance in a eukaryotic chromosome. EMBO J 13: 3395-3400.

Mendez J, Zou-Yang XH, Kim SY, Hidaka M, Tansey WP, Stillman B. 2002. Human origin recognition complex large 
subunit is degraded by ubiquitin-mediated proteolysis after initiation of DNA replication. Mol Cell 9: 481-491.

Merrick CJ, Jackson D, Diffley JF. 2004. Visualization of altered replication dynamics after DNA damage in human cells. I Biol Chem 279: 20067-20075.

Nyberg KA, Michelson RJ, Putnam CW, Weinert TA. 2002. Toward maintaining the genome: DNA damage and replication checkpoints. Annu Rev Genet 36: 617-656.

Osborn AJ, Elledge SJ, Zou L. 2002. Checking on the fork: the DNA-replication stress-response pathway. Trends Cell Biol 12: $509-516$.

Pacek M, Tutter AV, Kubota Y, Takisawa H, Walter JC. 2006. Localization of MCM2-7, Cdc45, and GINS to the site of DNA unwinding during eukaryotic DNA replication. Mol Cell 21: 581-587.

Paulovich AG, Hartwell LH. 1995. A checkpoint regulates the rate of progression through $S$ phase in $S$. cerevisiae in response to DNA damage. Cell 82: 841-847.

Remus D, Beuron F, Tolun G, Griffith JD, Morris EP, Diffley JF. 2009. Concerted loading of Mcm2-7 double hexamers around DNA during DNA replication origin licensing. Cell 139: 719-730.

Ricke RM, Bielinsky AK. 2004. Mcm10 regulates the stability and chromatin association of DNA polymerase- $\alpha$. Mol Cell 16: $173-185$.

Saha S, Shan Y, Mesner LD, Hamlin JL. 2004. The promoter of the Chinese hamster ovary dihydrofolate reductase gene regulates the activity of the local origin and helps define its boundaries. Genes Dev 18: 397-410.

Santocanale C, Diffley JF. 1998. A Mec1- and Rad53-dependent checkpoint controls late-firing origins of DNA replication. Nature 395: 615-618.

Santocanale C, Sharma K, Diffley JF. 1999. Activation of dormant origins of DNA replication in budding yeast. Genes Dev 13: 2360-2364.

Sarkaria JN, Busby EC, Tibbetts RS, Roos P, Taya Y, Karnitz LM, Abraham RT. 1999. Inhibition of ATM and ATR kinase activities by the radiosensitizing agent, caffeine. Cancer Res 59: 4375-4382.

Sawyer SL, Cheng IH, Chai W, Tye BK. 2004. Mcm10 and Cdc45 cooperate in origin activation in Saccharomyces cerevisiae. J Mol Biol 340: 195-202.

Sclafani RA, Holzen TM. 2007. Cell cycle regulation of DNA replication. Annu Rev Genet 41: 237-280.

Seiler JA, Conti C, Syed A, Aladjem MI, Pommier Y. 2007. The intra-S-phase checkpoint affects both DNA replication initiation and elongation: single-cell and -DNA fiber analyses. Mol Cell Biol 27: 5806-5818.

Sequeira-Mendes J, Diaz-Uriarte R, Apedaile A, Huntley D, Brockdorff N, Gomez M. 2009. Transcription initiation activity sets replication origin efficiency in mammalian cells. PLoS Genet 5: e1000446. doi: 10.1371/journal.pgen. 1000446.

Shibahara K, Stillman B. 1999. Replication-dependent marking of DNA by PCNA facilitates CAF-1-coupled inheritance of chromatin. Cell 96: 575-585.

Shirahige K, Hori Y, Shiraishi K, Yamashita M, Takahashi K, Obuse C, Tsurimoto T, Yoshikawa H. 1998. Regulation of DNA-replication origins during cell-cycle progression. Nature 395: 618-621.

Sporbert A, Gahl A, Ankerhold R, Leonhardt H, Cardoso MC. 2002. DNA polymerase clamp shows little turnover at established replication sites but sequential de novo assembly at adjacent origin clusters. Mol Cell 10: 1355-1365.

Syljuasen RG, Sorensen CS, Hansen LT, Fugger K, Lundin C, Johansson F, Helleday T, Sehested M, Lukas J, Bartek J. 2005.
Inhibition of human Chk1 causes increased initiation of DNA replication, phosphorylation of ATR targets, and DNA breakage. Mol Cell Biol 25: 3553-3562.

Takara TJ, Bell SP. 2009. Putting two heads together to unwind DNA. Cell 139: 652-654.

Takeda DY, Dutta A. 2005. DNA replication and progression through S phase. Oncogene 24: 2827-2843.

Trinklein ND, Chen WC, Kingston RE, Myers RM. 2004. Transcriptional regulation and binding of heat shock factor 1 and heat shock factor 2 to 32 human heat shock genes during thermal stress and differentiation. Cell Stress Chaperones 9: 21-28.

Tsakraklides V, Bell SP. 2010. Dynamics of pre-replicative complex assembly. I Biol Chem 285: 9437-9443.

Williams TM, Medina F, Badano I, Hazan RB, Hutchinson J, Muller WJ, Chopra NG, Scherer PE, Pestell RG, Lisanti MP. 2004. Caveolin-1 gene disruption promotes mammary tumorigenesis and dramatically enhances lung metastasis in vivo. Role of Cav-1 in cell invasiveness and matrix metalloproteinase (MMP-2/9) secretion. I Biol Chem 279: 51630-51646.

Wohlschlegel JA, Dhar SK, Prokhorova TA, Dutta A, Walter JC. 2002. Xenopus Mcm10 binds to origins of DNA replication after Mcm2-7 and stimulates origin binding of Cdc45. Mol Cell 9: 233-240.

Zhu W, Ukomadu C, Jha S, Senga T, Dhar SK, Wohlschlegel JA, Nutt LK, Kornbluth S, Dutta A. 2007. Mcm10 and And-1/ CTF4 recruit DNA polymerase $\alpha$ to chromatin for initiation of DNA replication. Genes Dev 21: 2288-2299. 


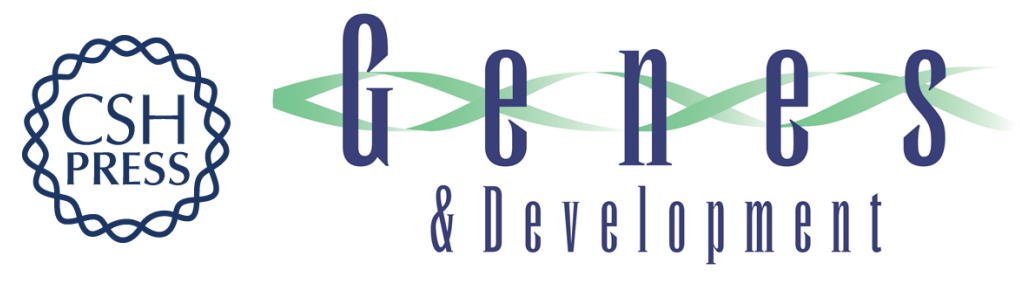

\section{The effect of the intra-S-phase checkpoint on origins of replication in human cells}

Neerja Karnani and Anindya Dutta

Genes Dev. 2011, 25:

Access the most recent version at doi:10.1101/gad.2029711

Supplemental http://genesdev.cshlp.org/content/suppl/2011/03/14/25.6.621.DC1
Material

References This article cites 63 articles, 27 of which can be accessed free at: http://genesdev.cshlp.org/content/25/6/621.full.html\#ref-list-1

License

Email Alerting

Service

Receive free email alerts when new articles cite this article - sign up in the box at the top right corner of the article or click here.

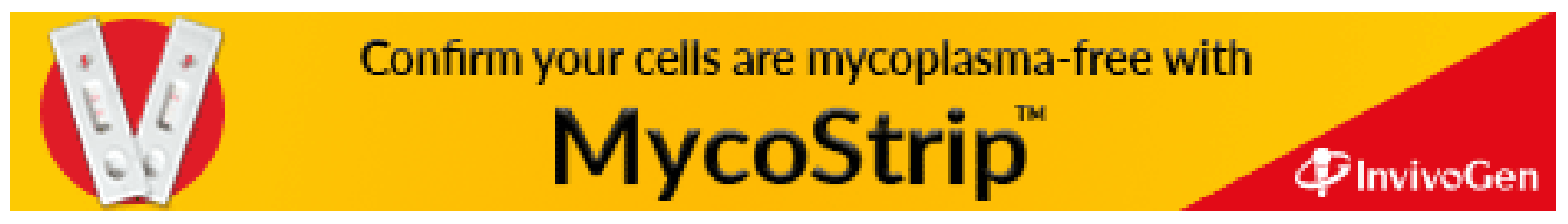

